

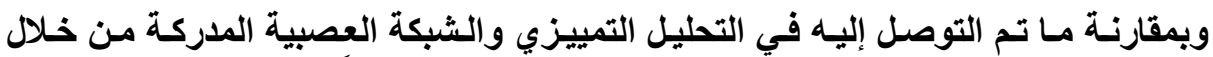

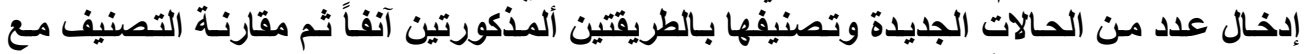

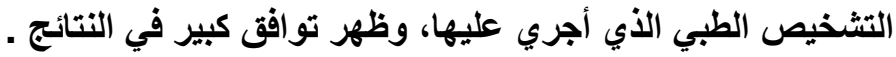

\title{
A Comparison of Discriminate Analysis, Neural Networks and Medical Diagnosis of Oral Cancer
}

\author{
Reem A. Al - Jaraah (PhD) \\ Lecture \\ Department of Dental Basic \\ Sciences
}

\author{
Manahil A. Azooz \\ Lecture Assist \\ Department of Dental Basic \\ Sciences \\ University of Mosul
}

\author{
Ali D. AL_Nuaimy \\ Lecture Assist \\ Deparment of Oral and \\ Miaxlofacial Surgery
}

\begin{abstract}
The current research aims at comparing the classification of: the discriminate analysis and the neural networks as a technique of computer sciences and artificial intelligence. Then comparing their results with the actual medical diagnosis of oral cancer that has been done on a sample consisted of (37) outpatient to Al - Jumhoori and Al - Salam Hospitals. In order to achieve the aims of the study, the data - base have been programmed via visual basic (v.6). The process includes all the medical data sheets for diagnosis (attached 1). In the discriminate analysis, three standards consisted of discriminate function. Finding the cut of point, the (rate of error) on the samples that includes (14), cases diagnosed medically (23), cases out of disease. The research demonstrated the discriminate function that can be used to distinct among with and without infected through knowing, cut of point amounted (-21.3), additionally knowing the rate of error referred in the end of the research that give a power of the discriminate function. A simplified perception neural network has been used as a successful procedure in classifying the cases of infected and non infected patients. By using $(\theta=0.75$, $\&(\eta=0.020)$ as a threshold value and training rate respectively. In comparing what has been done in discriminate analysis and neural networks perception via input a number new cases then classifying them according to the medical diagnosis done, showed a great coincidence in results

في الآونة الأخيرة.. اتجهت البحوث إلى معالجـة المشاكل قيد الدر اسـة بأسلوب

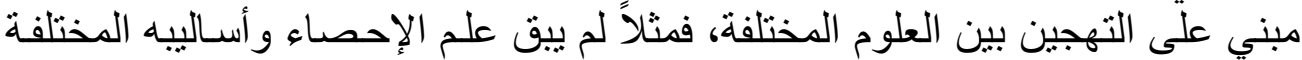

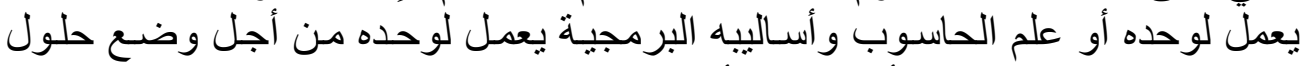

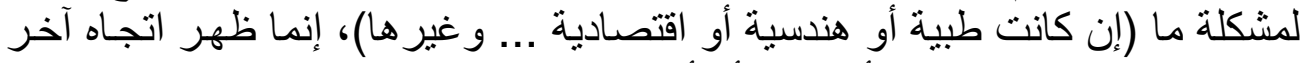

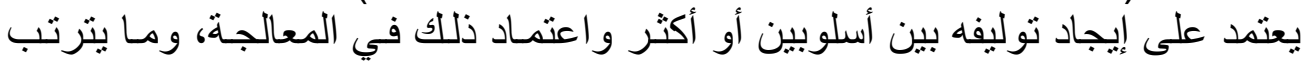

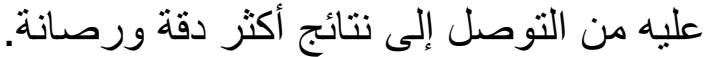

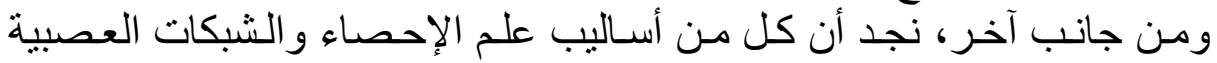

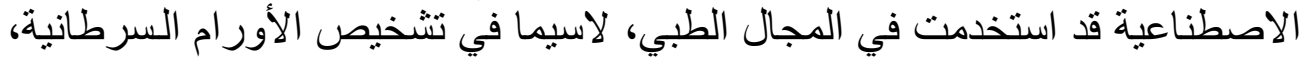

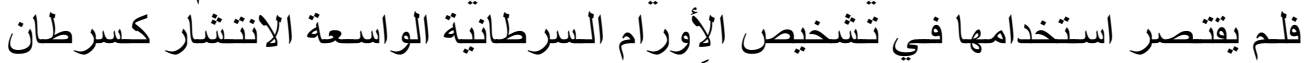

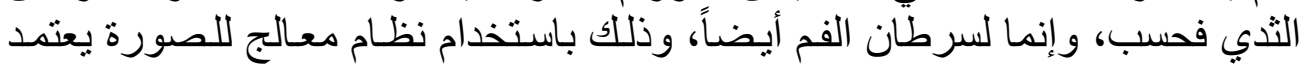
على استخدام شبكات عصبية للكثف عن الخلايا السرطانية من بين الخين الخايـا الطبيعيـة
\end{abstract}




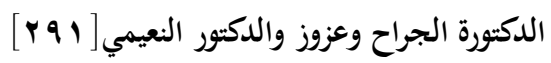

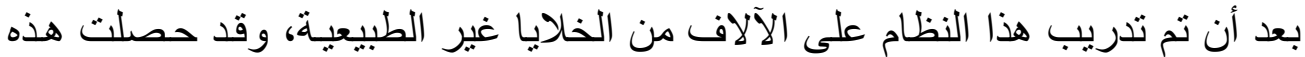

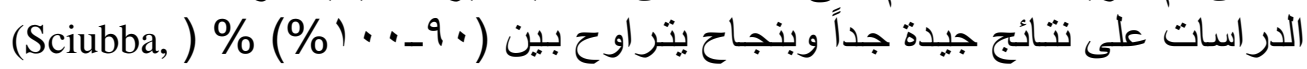
.(1999), (srirsky, et.al , 2002), Christian,2002 لقد قسم الجانب النظري لهذا البحث على محاور ثناثة هي:

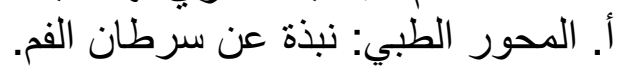
ب. المحور الإحصائي: التحليل التمييزي. ج. المحور البرمجي: الثبكة العصبية المدركة. ثم الجانب العملي الذي فيهـ نتائج التشخيص الطئية الطبي، التحليل التمييزي و الشبكة

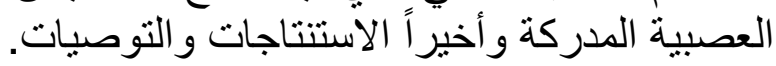

\section{اـ الجانب النظري \\ 1-1 الجحور الطبي- نبذة عن سرطان الفم}

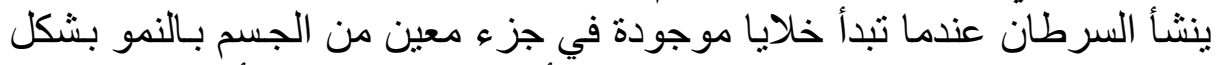

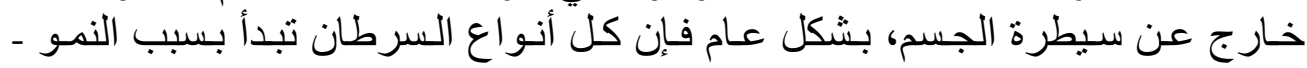
الخارج عن البيطرة ـ لخلايا غير طبيعية (American Cancer Society, 2004).

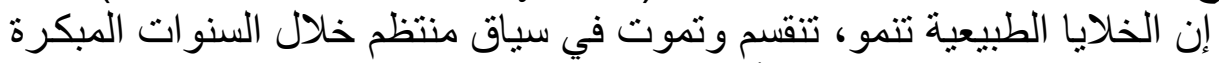

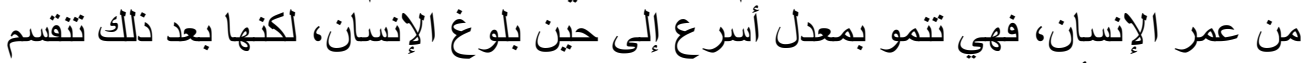

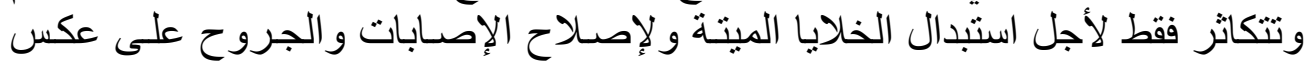
الخلايا السرطانية التي تستمر بالنمو والانقسام مكونة خلايا سرطانية الإنية جديدة.

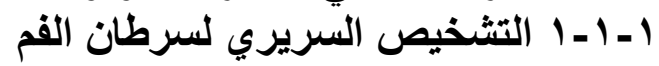

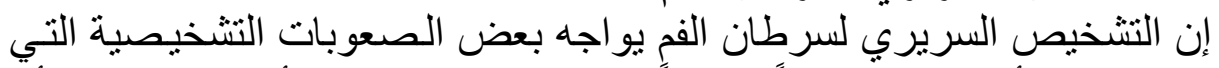

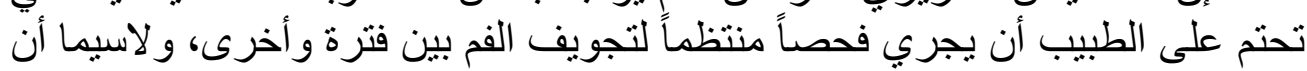

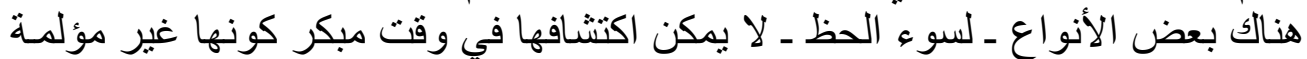

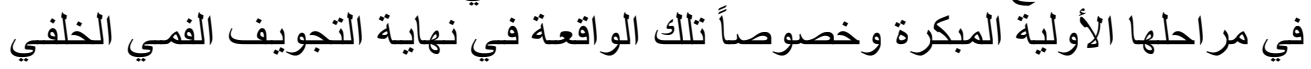

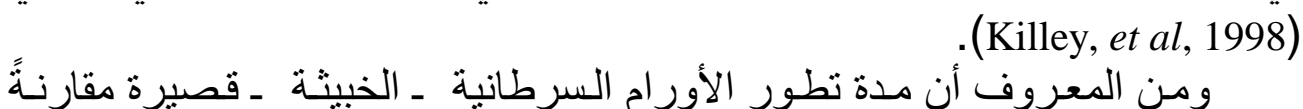

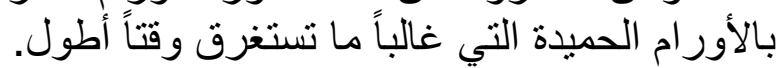

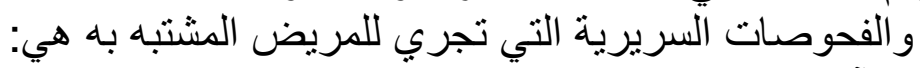

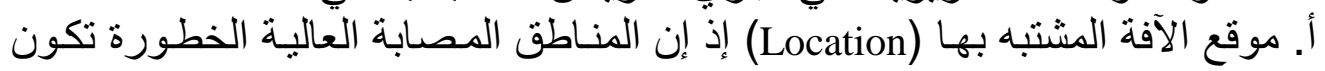

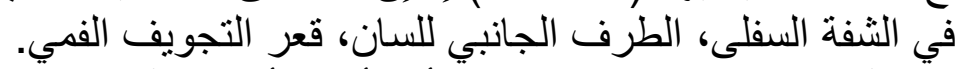
ب. لون الآفة المشتبه بها (Color)، إذ تأخذ أحد الألوان الآتية:

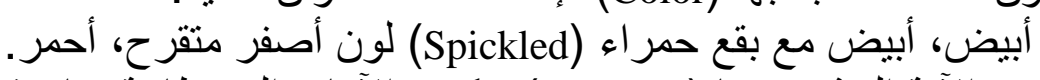
ج. عمر الآفة المشتبه بها (Duration)، تكون الآفات السرطانية عادة قصيرة العمر وسريعة التطور.

د. عمر المصاب بالآفة المشتبه بها (Age)، إذ غالباً تصيب الآفات السرطانية الفمويـة

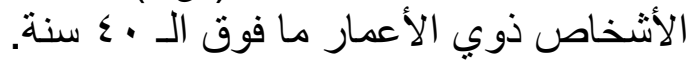




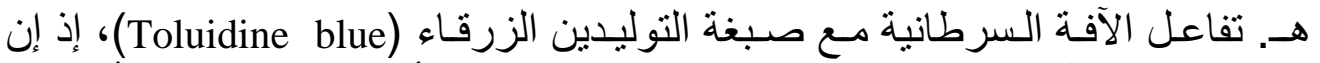

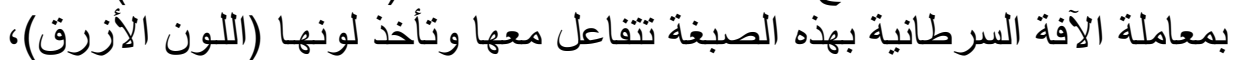

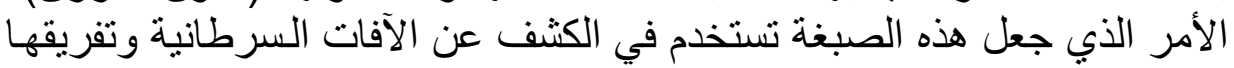
عن الآفات غير السرطانية.

\section{1 ـ ا - Y التشخيص المختبري لسرطان الفم}

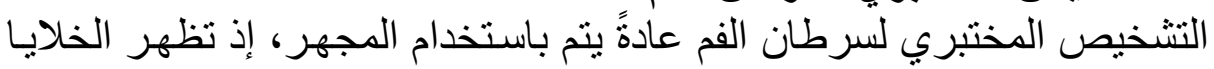

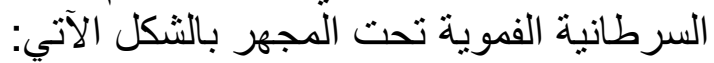
أ. تجمعات خلوية ذات نوى كبيرة عالية الكروماتيز باتين وسايتو بلازم خفيف. ب. عدم انتظام صبغة الكروماتين. ت. تمتلك الخلايا السرطانية نويات كبيرة النيات ومتعددة.

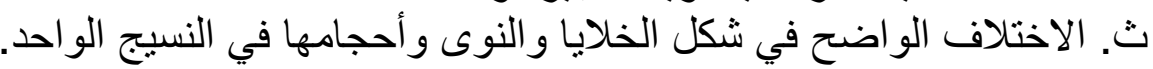
ج. زيادة عالية وملحوظة في معدل انقسامات الخلايا. (Cawson, et.al, 2002).

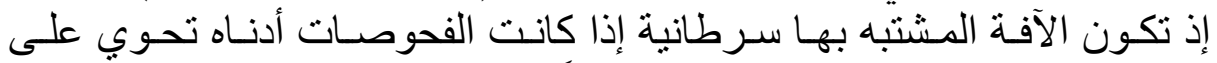

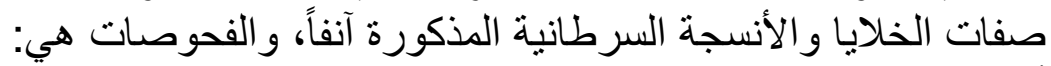
أ. فحص الانقشار الخلوي (Exfoliative Cytology). ب. طريقة الرشف الخلوي بالإبرة الدقيقة (Fine Needle Aspiration). ت. طريقة أخذ مقطع أو جزء من الرئ الآفة (Incisional Biopsy). ث. طريقة إزالة الآفة كلياً (Excisional Biopsy).

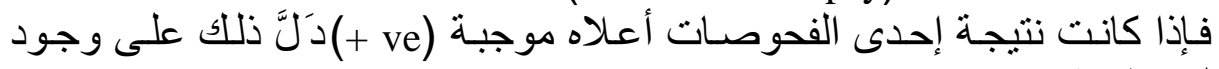
الآفة السرطانية.

\section{ا - r المحور الإحصائي: التحليل التمييزي}

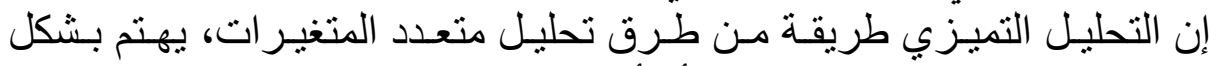

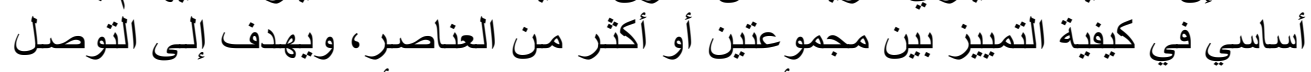

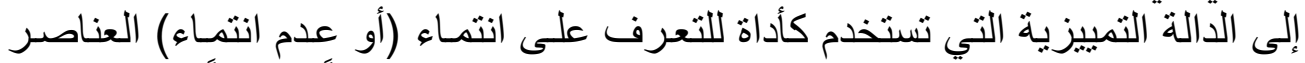

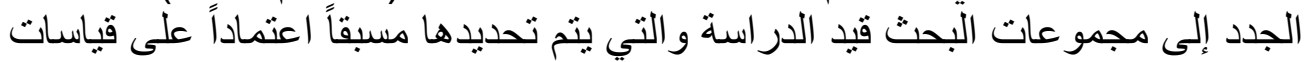

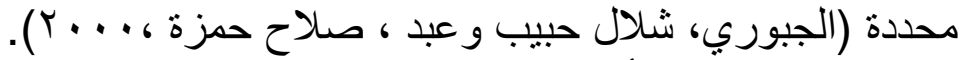

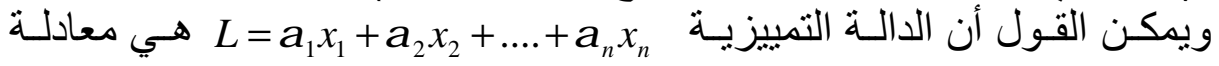

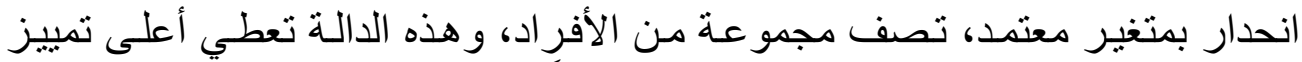

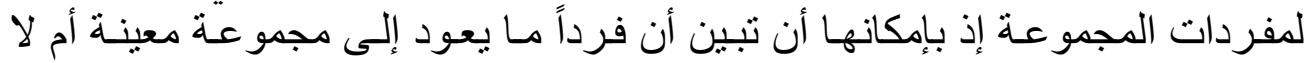
.(Kleinbaum \& Kupper, 1988)

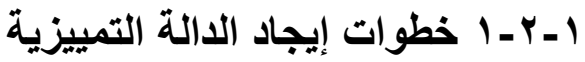
ا ـ حساب di d الفروقات بين متوسطي كل متغير في المجمو عتين. 
الدكتورة الجراح وعزوز والدكتور النعيمي[بح [ب]

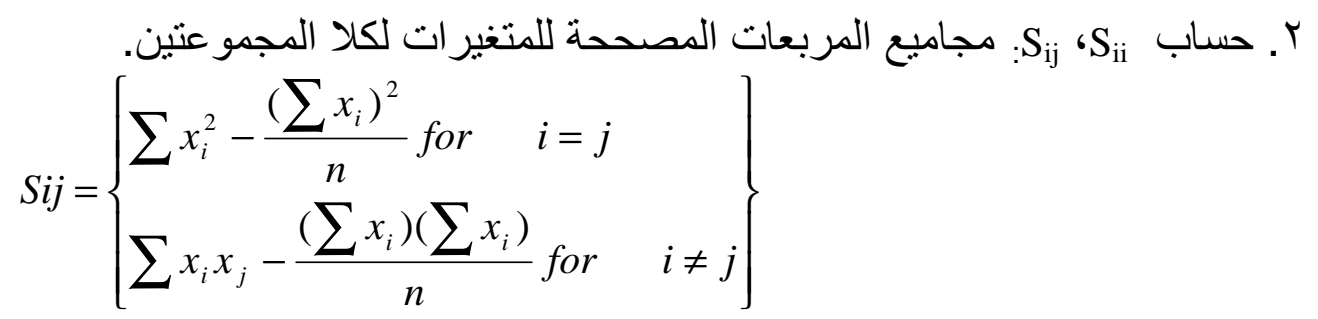

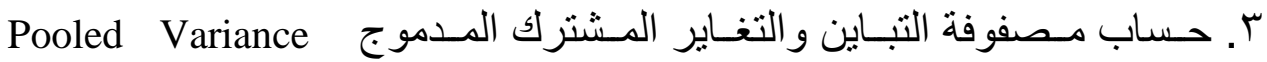
$V i j=\left\{\begin{array}{ll}{\left[S_{i i(1)}+s_{i i(2)}\right] /\left(n_{1}+n_{2}-2\right) \text { for }} & i=j[ \\ {\left[S_{i j(1)}+s_{i j(2)}\right] /\left(n_{1}+n_{2}-2\right) \text { for }} & i \neq j\end{array}\right\}$

covariance matrix

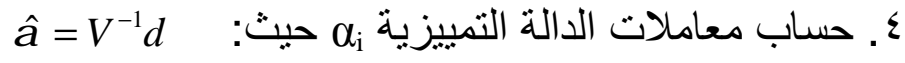

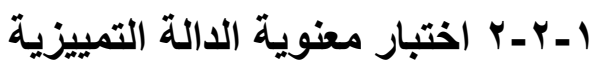

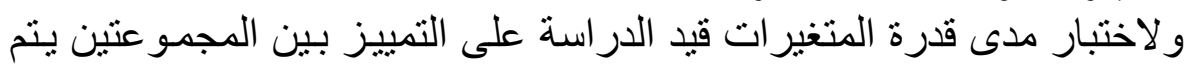
إيجاد قيمة المختبر الإحصائي F حيث:

$$
\begin{aligned}
& F=\left[\left(n_{1} \cdot n_{2}\right)\left(n_{1}+n_{2}-m-1\right) /\left(n_{1}+n_{2}\right)\left(n_{1}+n_{2}-2\right) m\right] * D^{2} \\
& D^{2}=d^{\prime} \hat{\alpha}
\end{aligned}
$$

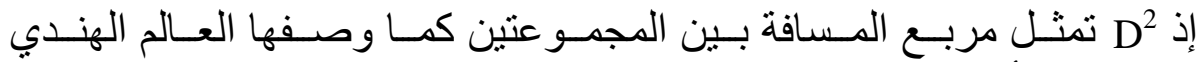

Mahalanobise

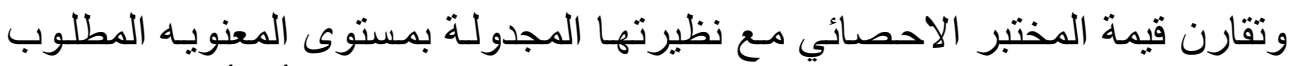
ودرجـة الحريـة (m,

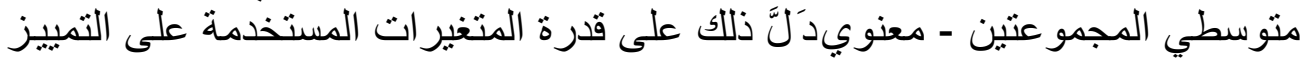
بين المجمو عتين (Anderson,1984) و (Dillon \& Goldstein, 1984) .

\section{Cut off Point}

لمعرفة الحد الفاصل بين المجمو عتين عند تطبيق الدالة التمييزية على الحالات المستقبلية يتم احتساب نقطة الفصل، وكما يأتي : الفين الهن 1 ـ . إيجاد متوسط المجمو عتين:

$$
\begin{gathered}
\bar{L}(1)=\hat{\alpha}_{1} \bar{x}_{1}(1)+\hat{\alpha}_{2} \bar{x}_{2}(1)+\ldots+\hat{\alpha}_{n} \bar{x}_{n}(1) \\
\bar{L}(2)=\hat{\alpha}_{1} \bar{x}_{1}(2)+\hat{\alpha}_{2} \bar{x}_{2}(2)+\ldots+\hat{\alpha}_{n} \bar{x}_{n}(2) \\
L c=(\bar{L}(1)+\bar{L}(2)) / 2
\end{gathered}
$$
r ـ ـ أساب Lc: نقطة الفصل حيث:

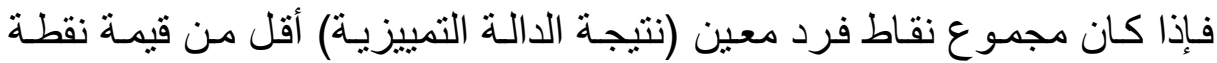

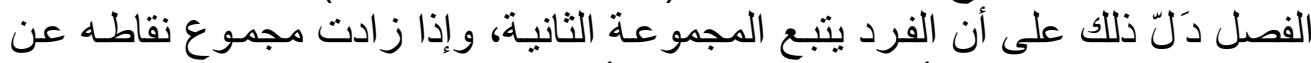

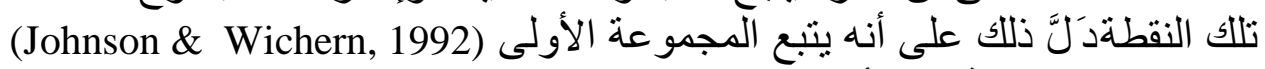




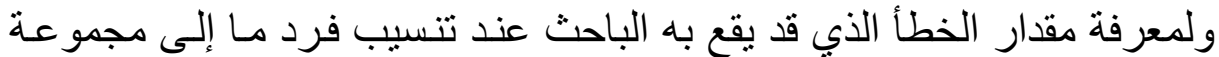

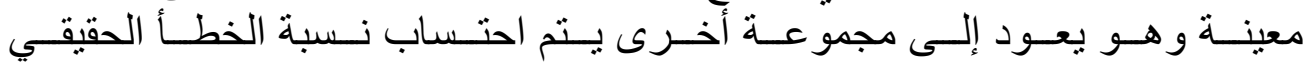
True error rate $=f\left(-\sqrt{D^{2}} / 2\right)$

و هو عبارة عن المساحة تحت المنحني الطبيعي، تستخرج جئي من جدول مساحات

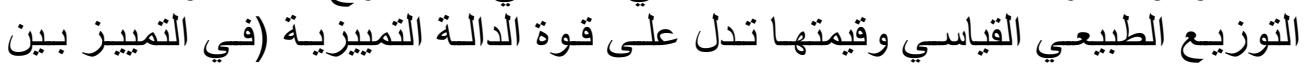
المجاميع) و هي ايجابية كلما كانت ذات قيمة صغيرة (Kleinbaum \& Kupper,1988).

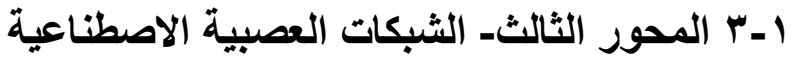

نتيجة لتطور الثبكات العصبية الاصنية الصناعية أصبح بمقدور ها أداء واء وظائف تثنابه

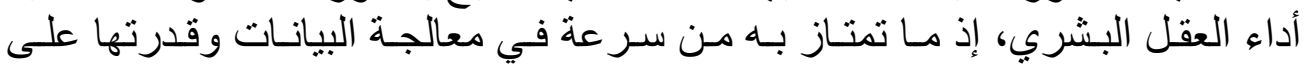

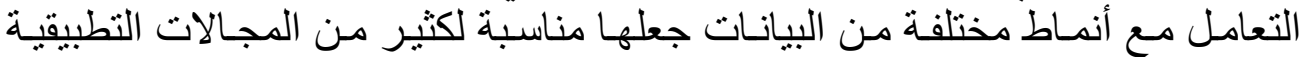

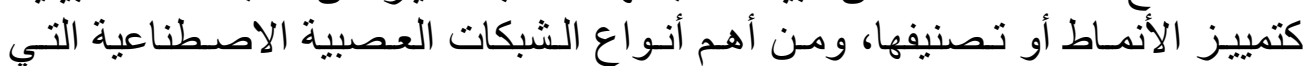
تستخدم لهذا الغرض هي الثبكة العصبية الددركة (Shiying \& Yanhua, 1998).

\section{Perceptron Neural Network 1-1 الثبكة العصبية المدركة}

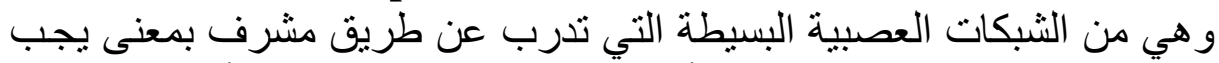

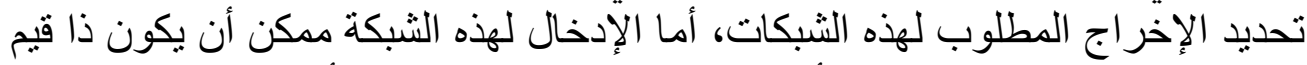

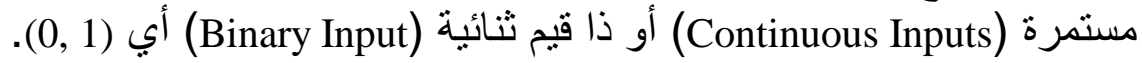
و هذه الثبكة تستخدم دالة تنشيط مناسبة لتحديد مستوى الفعالية للخلية التية العصبية،

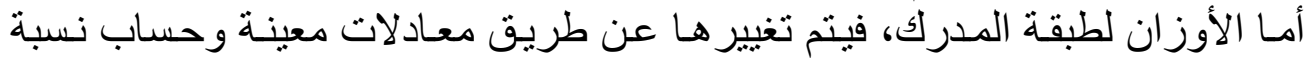

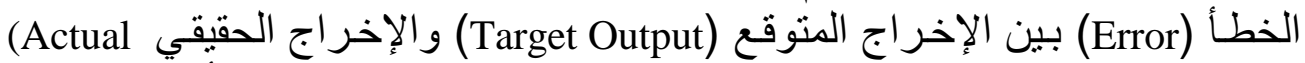

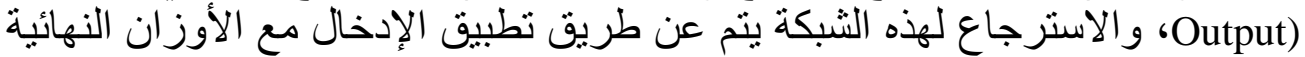
الناتجة من تدريب الثبكة لنحصل على الإخر اج المطلوب (Wasserman,1989).

\section{r-r-1}

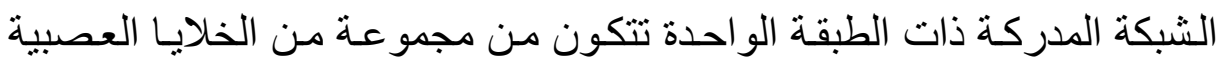

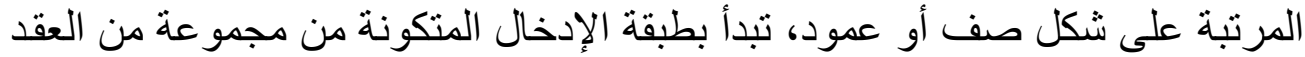

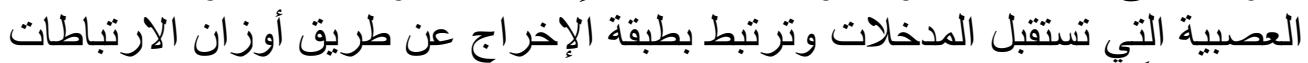

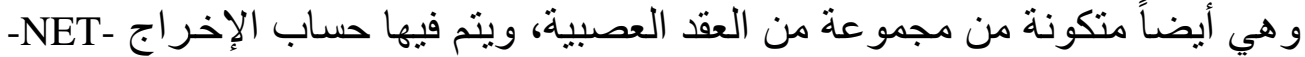

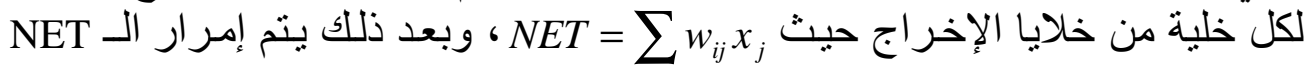

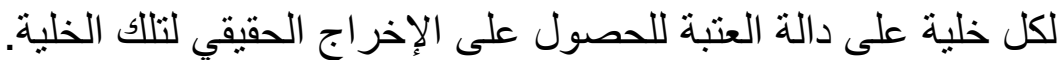

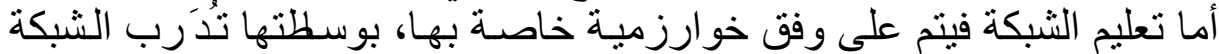

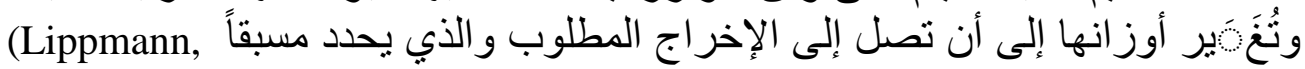


الدكتورة الجراح وعزوز والدكتور النعيمي[90]

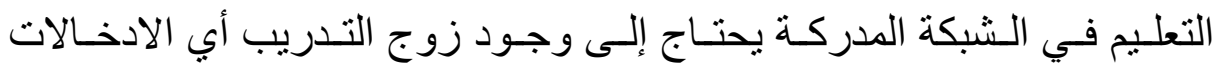

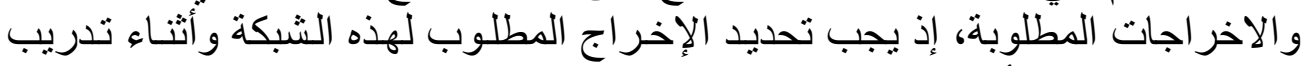

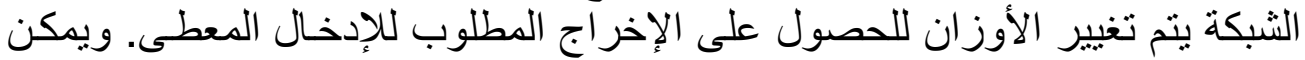
تلخيص خوارزمية التعليم للشبكة المدركة بالخطوات الاترات الآتية:

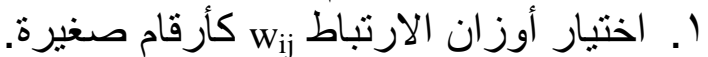

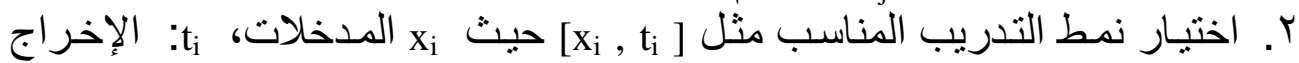

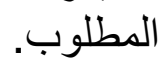

$$
\begin{aligned}
& W_{i j}^{\text {new }}=W_{i j}^{\text {old }}+\Delta W_{i j} \\
& \Delta W_{i j}=\eta \cdot e_{i} \cdot E_{i} \\
& E_{i}=t_{i}-f\left(\sum W_{i k} X_{k}\right)
\end{aligned}
$$

ـــ الرجوع إلى الخطوة الثانية.

$$
\text { r. الجانب العليث }
$$

شمل البحث (rV (T) مريضاً من المر اجعين إلى كل من المستشفى الجمهوري

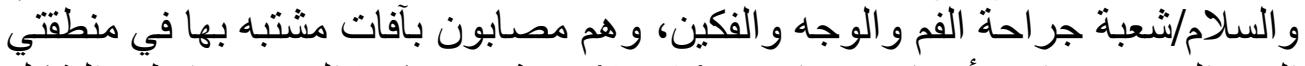

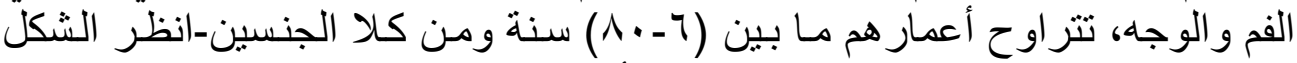

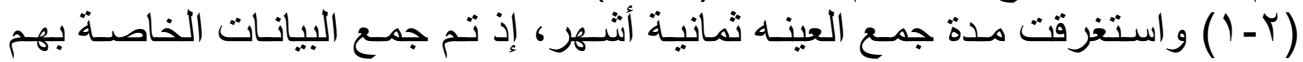

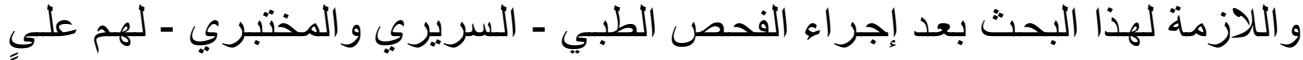

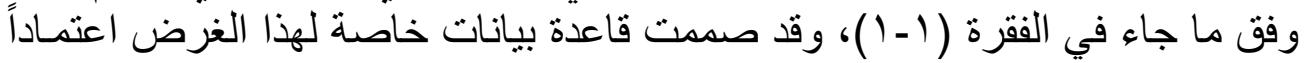
على نظام فجو ال بيسك وربطها بنظام Access (ملحق ل (). 


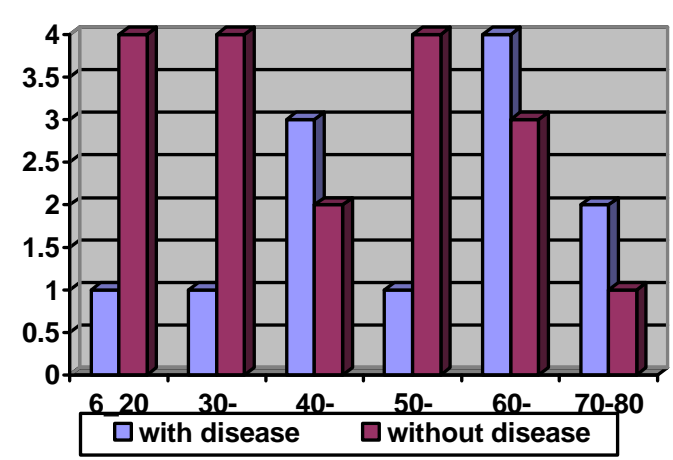

شكل (b-1-2): العينة حسب العمر

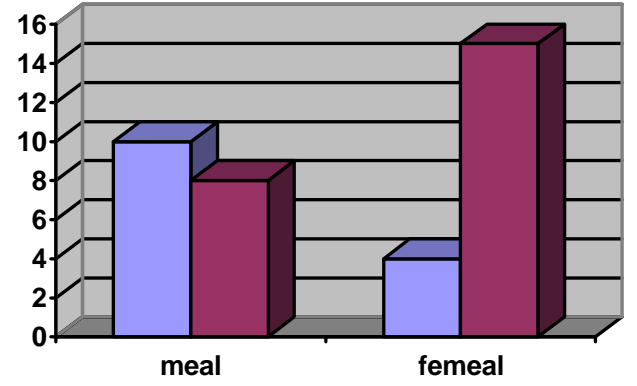

$\square$ with disease $\quad \square$ without disease

توزيع عينة البحث حسب الجنس والعمر

\section{ب - ا ـ ا 1 متغيرات البحث}

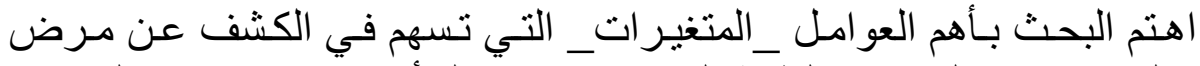
سرطان الفم، وحسب المصادر الطبية المعتمدة في هذا الثأن، وكما جـاء في الفئه الفقرتين

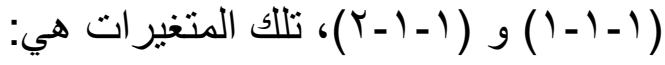

\begin{tabular}{|c|c|c|c|}
\hline $\mathrm{X}_{6}$ & شكل الآفة المشتبه بها & $\mathrm{X}_{1}$ & فحص مقطع من الآفة \\
\hline $\mathrm{X}_{7}$ & لون الآفة & $\mathrm{X}_{2}$ & فحص الرشف الخلوي بالإبرة الدقيقة \\
\hline $\mathrm{X}_{8}$ & موقع الآفة & $\mathrm{X}_{3}$ & فحص الانتشار الخلوي \\
\hline$X_{9}$ & عمر المريض & $\mathrm{X}_{4}$ & تفاعل الآفة مع \\
\hline 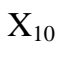 & جنس المريض & $\mathrm{X}_{5}$ & عمر الآفة المشتبهـ بها \\
\hline
\end{tabular}

تم إجر اء الفحوصبات الطبي للمرض السريرية اللازمة لكل مريض، إذ أُخضعت كل آفة مشتبه

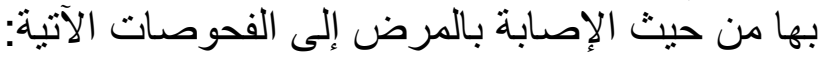

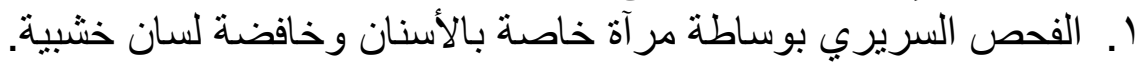

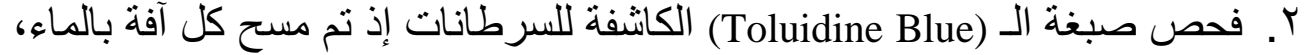

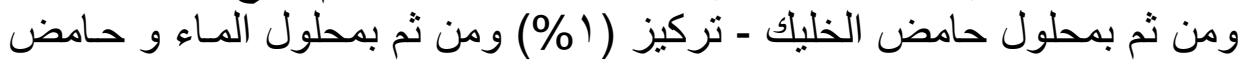
الخليك مرة أخرى لإز الة الصبغة الز ائدة.

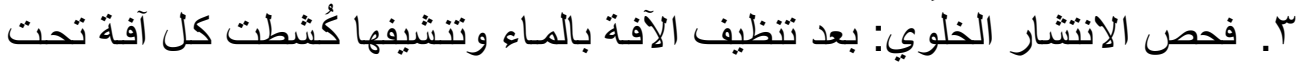

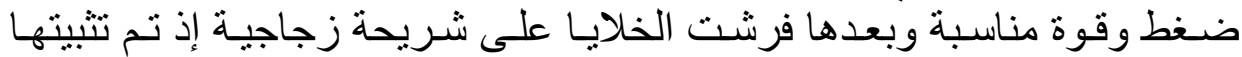

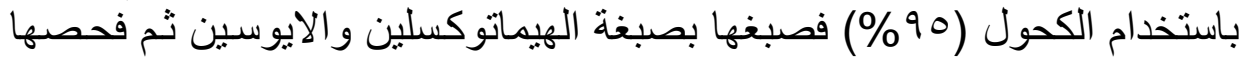




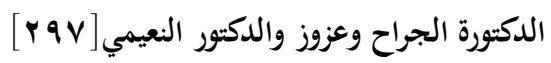

ع. فحص الرشف الخلوي بـالإبرة الدقيقة: بعد تنظيف الآفة بالمساء وتنشيفها أُدخلت

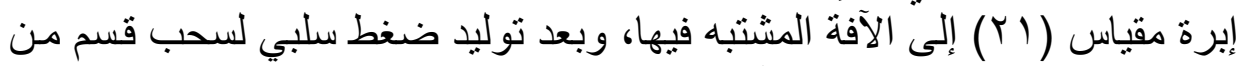

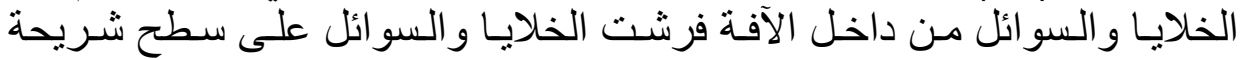
زجاجية وعوملت كما في الفحص ألعاب الألاه.

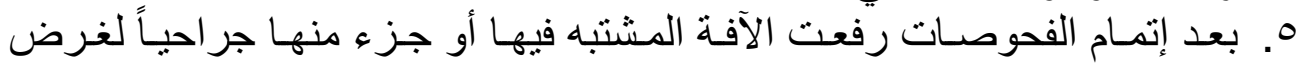

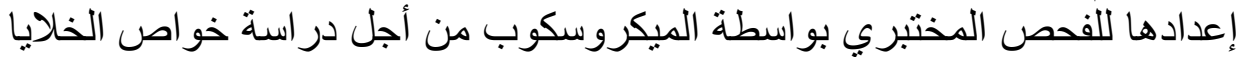
و الأنسجة مجهريا وتثخيص الأنسجة السرطانية من غير السرطانية.

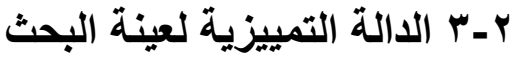

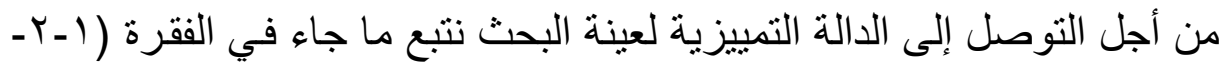

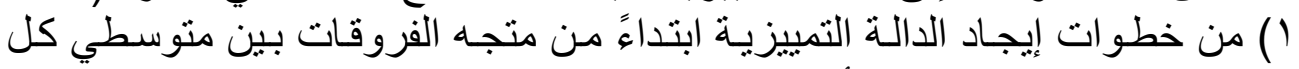

\begin{tabular}{|c|c|c|c|c|c|c|c|c|c|}
\hline$d_{1}$ & $d_{2}$ & $D_{3}$ & $d_{4}$ & $d_{5}$ & $d_{6}$ & $d_{7}$ & $d_{8}$ & d \\
\hline-1.77 & -1.86 & -1.714 & -0.32 & -0.87 & -131.8 & 0.2112 & 0.171 & -17.43 & 0.339 \\
\hline
\end{tabular}

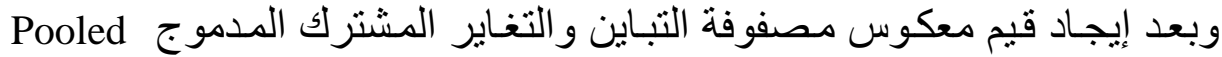
Var-Cov.

\begin{tabular}{|c|c|c|c|c|c|c|c|c|c|}
\hline$\alpha^{\wedge}{ }_{1}$ & $\alpha^{\wedge}{ }_{2}$ & $\alpha^{\wedge}{ }_{3}$ & $\alpha^{\wedge}{ }_{4}$ & $\mathrm{~A}^{\wedge} 5$ & $\alpha^{\wedge}{ }_{6}$ & $\alpha^{\wedge}{ }_{7}$ & $\alpha^{\wedge} 8$ & $\alpha^{\wedge}{ }_{9}$ & $\alpha^{\wedge} 10$ \\
\hline-7.991 & -22.41 & 1.347 & 1.346 & -10.96 & 0.0036 & -0.262 & -1.15 & -0.115 & 1.4991 \\
\hline
\end{tabular}

و عليه فان دالة التمييز هي:

$\mathrm{L}=-7.991 \mathrm{X}_{1}-22.412 \mathrm{X}_{2}+1.347 \mathrm{X}_{3}+1.346 \mathrm{X}_{4}-10.96 \mathrm{X}_{5}+0.0036 \mathrm{X}_{6}-$ $0.262 \mathrm{X}_{7}-1.15 \mathrm{X}_{8}-0.115 \mathrm{X}_{9}+1.4991 \mathrm{X}_{10}$

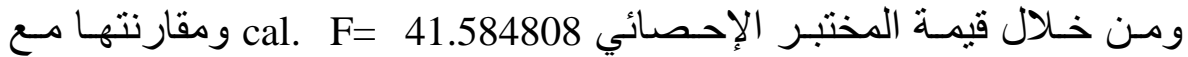

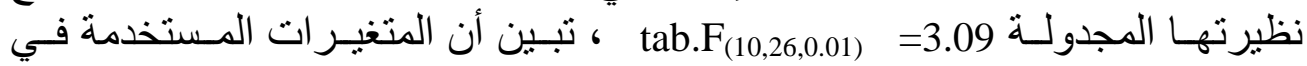
المجمو عتين قادرة على التمييز بين المصابين بالمرض المن من عدمه.

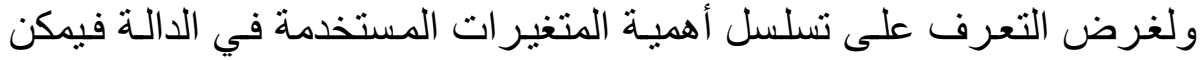
تطبيـق الصيغة:

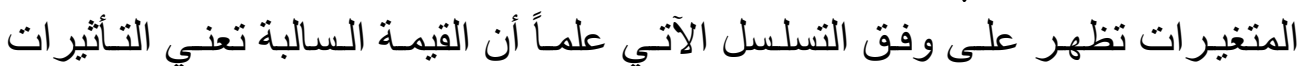

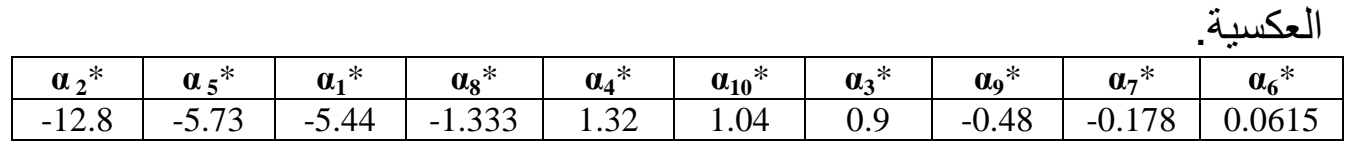

أما نقطة الفصل بين المجمو عتين فهي (LC=-21.3) التي نم الحصول عليها بعد

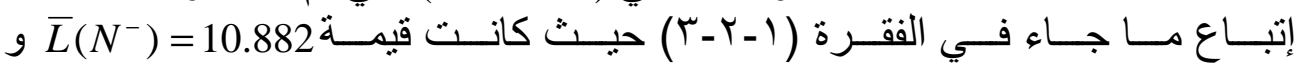
$\bar{L}\left(P^{+}\right)=-53.468$ 
أخير اً فإن نسبة الخطأ (الاحتمال) الذي وقع فيه الباحثون بلغ القيمـة (0.0003)،

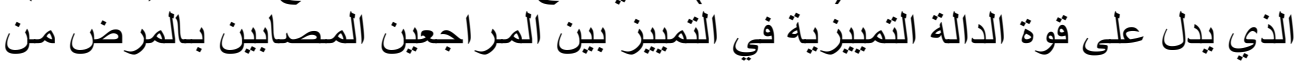

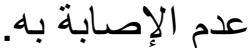

r ـ ـ الثبكة العصبية المدركة لتشخيص سرطان الفم

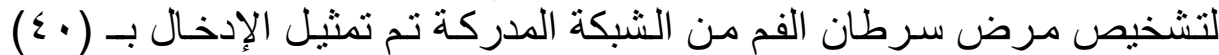

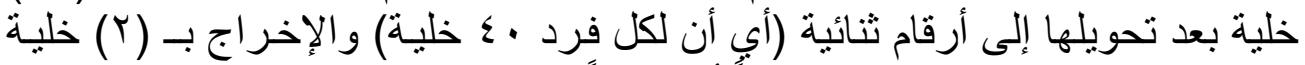

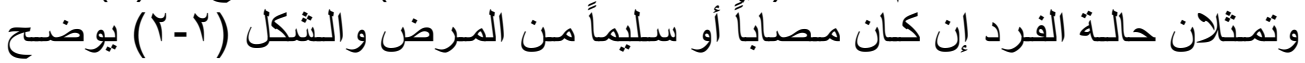
معمارية الشبكة العصبية المستخدمة في تشخيص مرض مرض سرطان سنان الفم.

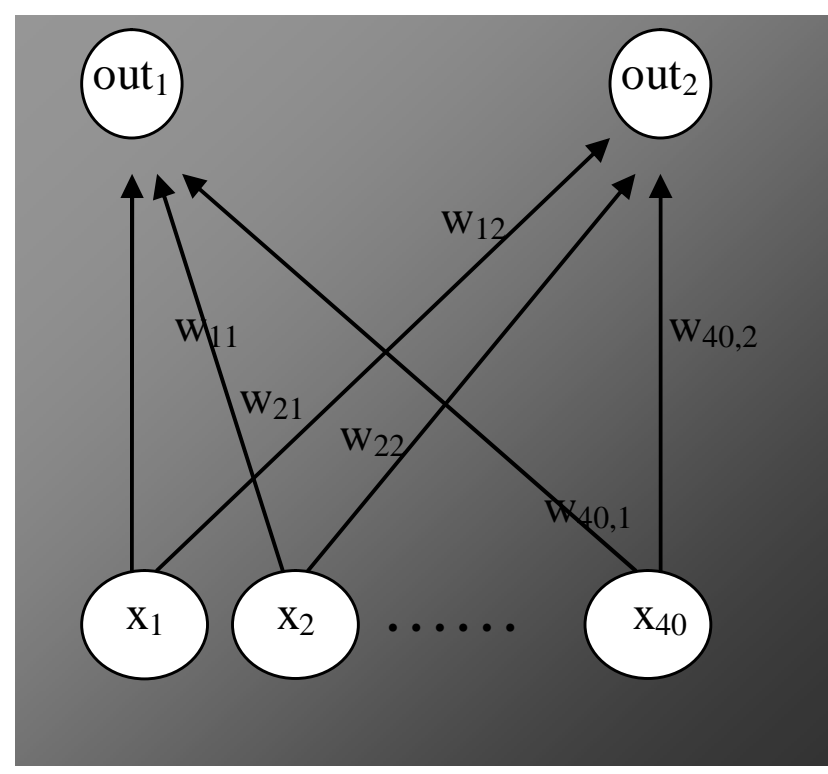

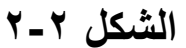

معمارية الثبكة العصبية المستخدمة في تثخيص مرض سرطان الفم

وقد وضعت للشبكة أعلاه خو ارزمية لتدريبها على الأنماط التي تم إدخالها في

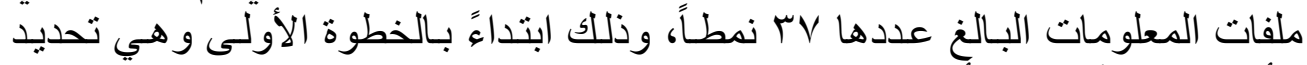

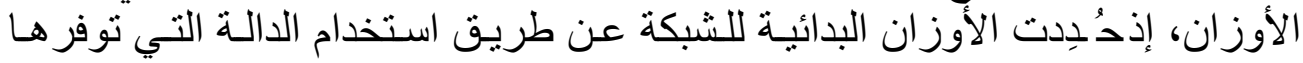

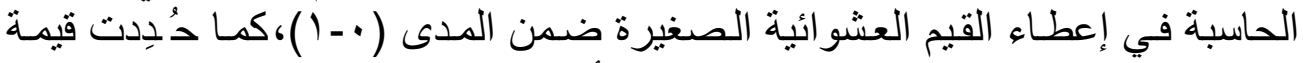

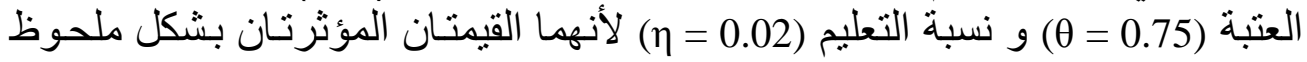

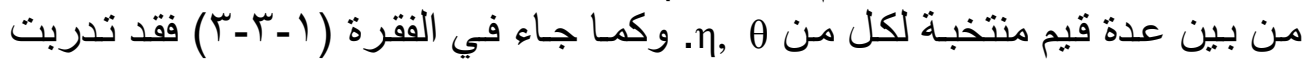

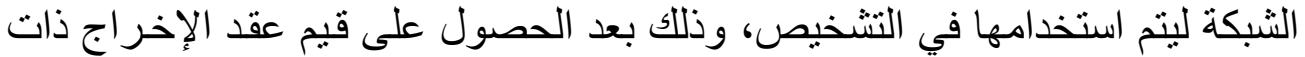

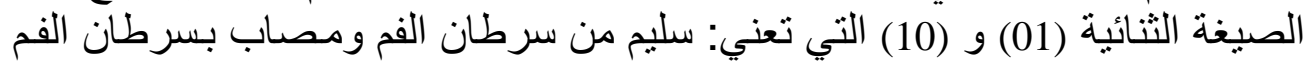
على التو الي. 
ومن أجل التعرف على مقدار التطابق بين ما تم التوصل إليهه من نتائج التمييز

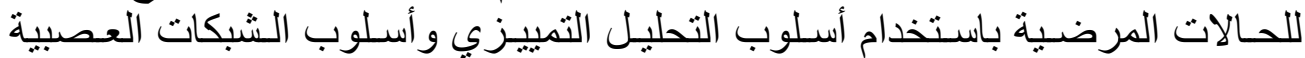
الاصطناعية ومقارنتها بالتشخيص الطبي، تم تطبيق بيانات لخمسة مشاهدات وكانت

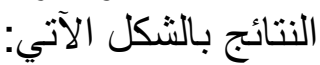

\begin{tabular}{|c|c|c|c|c|}
\hline النتيجة & الثبكة العصبية & قيمة دالة التحليل & التشخيص & المشاهدة \\
\hline مطابق & 10 & -36.41 & $\mathrm{P}^{+}$ & الأولى \\
\hline غير مطابق & 01 & -20.09 & $\mathrm{P}^{+}$ & الثانية \\
\hline مطابق & 01 & 5.8 & $\mathrm{~N}^{-}$ & الثالثة \\
\hline مطابق & 10 & -41.08 & $\mathrm{P}^{+}$ & الر ابعة \\
\hline مطابق & 01 & 7.3 & $\mathrm{~N}^{-}$ & الخامسة \\
\hline
\end{tabular}

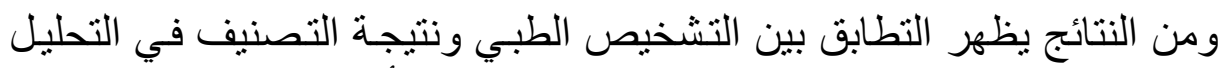

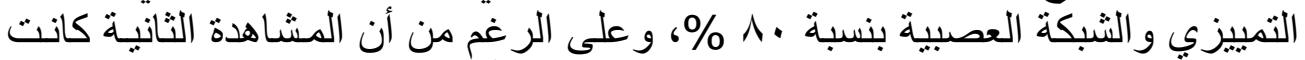

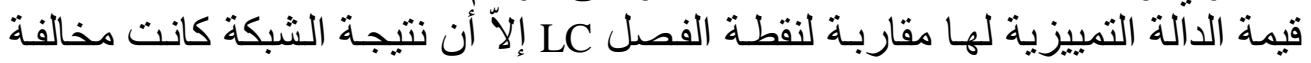
للتشخيص الطبي، و هذا يعود إلى المعلومات المستقاة من المشاهدة نفسها.

$$
\text { ع. الاستتتاجات }
$$

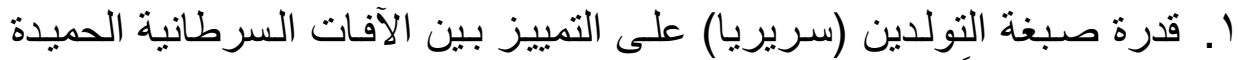

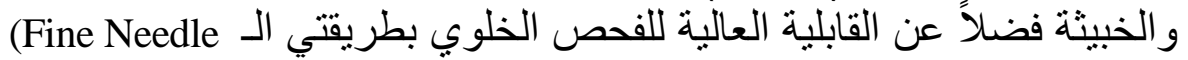

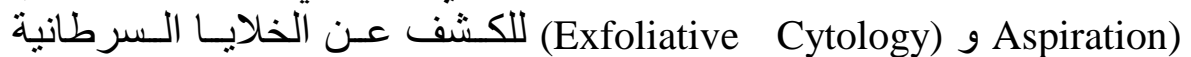
الخبيثة من بين الخلايا الطبيعية غير المصابة.

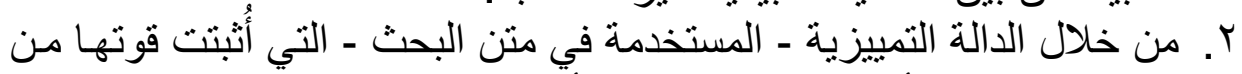

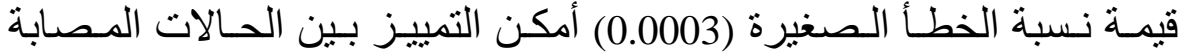

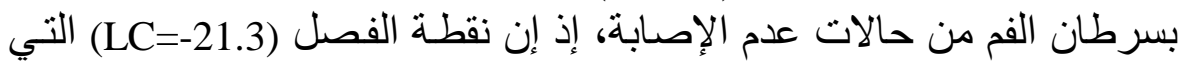

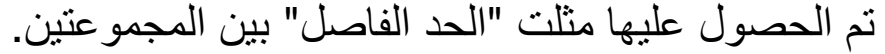

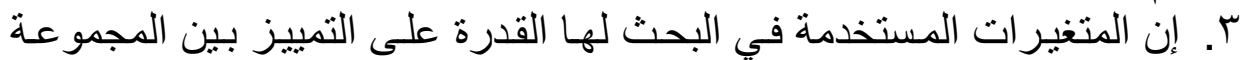

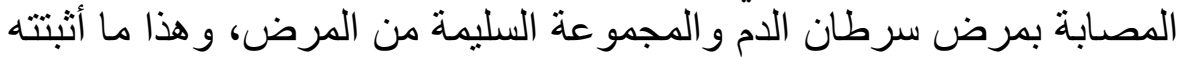

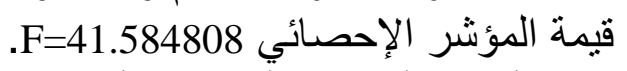

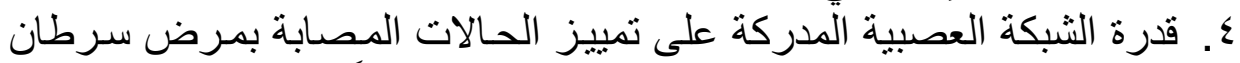

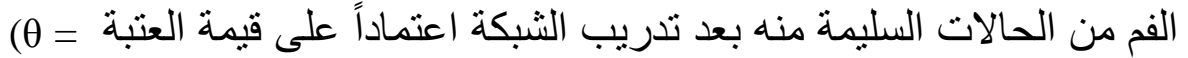

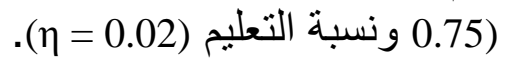

0. التو افق في نتيجة التمبيز باستخدام الدالة التمبيزيـة و الثبكة العصبية المدركة

$$
\begin{aligned}
& \text { مع التشخيص الطبي للمرض في لـ لـ حدٍ كبير. } \\
& \text { 7. و ونالك نتيجة عرضية من العينة هي: }
\end{aligned}
$$




\section{$[r \cdot . \cdot]$}

مقارنة بين التحليل التمييزي...

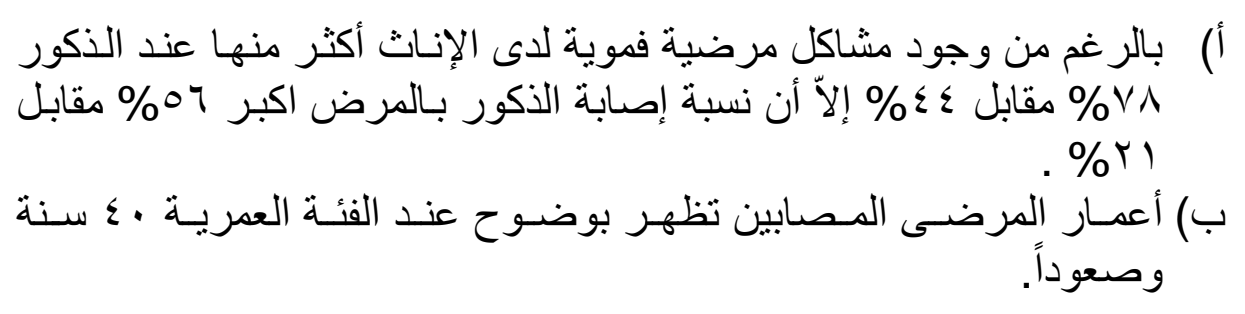

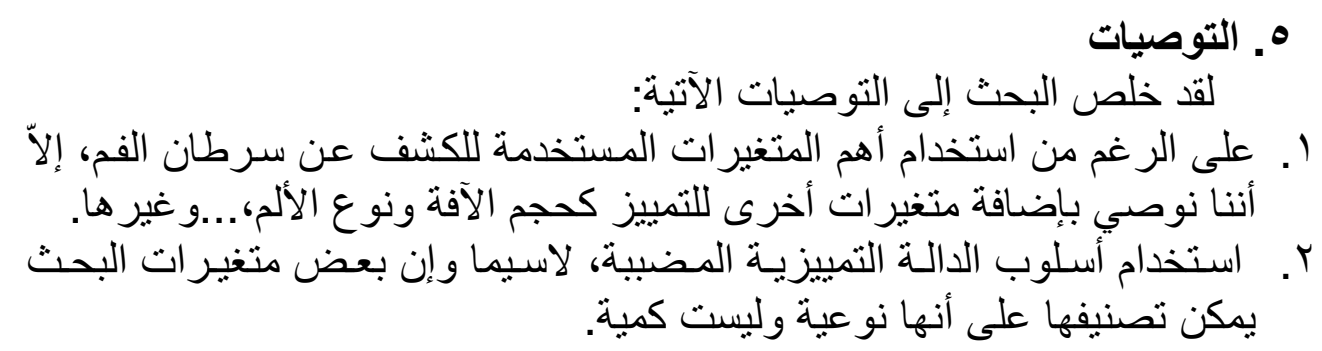

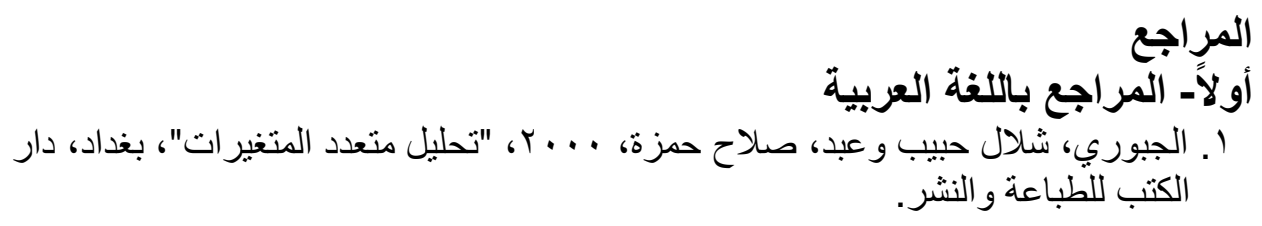

ثانياً. المراجع باللغة الاجنبية

1. Anderson T.W., 1984: An Introduction to Multivariate Statistical Analysis, $2^{\text {nd }}$ Edition, John Wiley \& Sons.

2. Cawson R.A., Odell E.W. \& Porter S., 2002: Principles of investigation and diagnosis, $\mathrm{Pq}$ in Essentials of oral Pathology and Oral Medicine, $7^{\text {th }}$ edition, London Churchill Livingstone Publications.

3. Christian D.C., 2002: Computer-assisted analysis of oral brush biopsies at an oral cancer screening program. J.A.D.A.,133,357-362.

4. Dillon W. \& Goldstein M., 1984: Multivariate Analysis, Methods \& Applications, John Wiley \& Sons.

5. Johnson R. and Wichern D., 1992. Applied Multivariate Statistical Methods, $3^{\text {rd }}$ Edition, Prentice Hall.

6. Killey H.C., Seward G.R. and Key L.W., 1998: Some additional examination carried out when investigating lesion, Outline of oral surgery, part 2.

7. Kleinbaum, D. J. \& Kupper, L. L., 1988: Applied Regression Analysis and Other Multivariate Methods. Massachusetts Duxbury press.

8. Lipmann R.P., 1987: Introduction to computing with Neural Nets, IEEE Assp Magazine, Vol.4, No.2.

9. Sciubba J., 1999: Improving detection of precancerous and cancerous oral lesions Computer assisted analysis of the oral brush biopsy, J.A.D.A., 130: 1445-1457.

10. Shiying H. \& Yanhua Z., 1998: Kohonen Clustering Networks with fuzzy Selective Multi resolution for intensity Image Segmentation ,International conference on Neural Networks and Brain proceeding, china.

11. Svirsky J.A., Burns J.C. ,Carpenter W.M., Cohen D.M., Battachayya I \& Fautasia J.E., 2002: Comparison of computer-assisted brush biopsy results with follow up biopsy and history ,Gen. Dent., 50,500-503. 
الدكتورة الجراح وعزوز والدكتور النعيمي[ ( إ]

12. Wasserman P.D., 1989: Neural computing theory and practice, Van Nostrand Reinhold, New York. 